\title{
Mitteilungen des Arbeitskreises der Chefärzte und Chef- ärztinnen von Kliniken für Psychiatrie und Psychotherapie an Allgemeinkrankenhäusern in Deutschland (ackpa)
}

Verantwortlich für diese Rubrik: Karl H. Beine, Hamm

\section{Tagungsbericht 59. Kommende- Treffen der ackpa am 21.9.2015 in Kassel \\ $\nabla$}

Dr. Ingrid Munk begrüßt die Teilnehmer, mit herzlichem Dank an Prof. Martin Ohlmeier für 13 Jahre Gastgeberschaft der Kommende beim Ludwig Noll Krankenhaus in Kassel. Drei Projekte werden am Vormittag vorgestellt.

Dr. Dr. Christopher Rommel, Treuenbrietzen: Die Funktion des Chefarztes und die Rolle der CA-Visite. „Welches Theater veranstalten wir in unseren Kliniken?" Eine Umfrage in seiner Klinik ergab heterogene Ergebnisse hinsichtlich der Erwartungen der Mitarbeiter, darunter einige Tendenzen: Die Mitarbeiter wünschen sich Klarheit bei Rechtsfragen, Beitrag zum Krankheitsverständnis einzelner Patienten, Klärung von Konflikten, Unsicherheiten beseitigen, mehr Zeit für Angehörige und Weiterbildungsinhalte. Vielfältige Visiten werden durchgeführt: Durch die Zimmer gehen (mit oder ohne Schwarm), Delegation, Kurvenvisite, Teilnahme an Supervision/Gruppentherapie/Therapiesitzung/ Teambesprechung, Einzelvisite, Visite in einer Blitzlichtgruppe, Besuch in der Kunsttherapie... Feedback von Patienten wird anhand von Fallvignetten vorgestellt, über Text und Bilder. Rommel arbeitet mit
Kurzinterventionen, Metaphern, Übungen und - machmal provokativ formulierten Anregungen. Empfohlen wird: An den eigenen Erwartungen orientieren, Authentizität als Vorbild für Assistenten und Mitarbeiter.

Dr. Martin Zinkler, Heidenheim, erläutert die Auswirkungen der UN-Behindertenrechtskonvention auf die psychiatrische Behandlung in unseren Kliniken und in den ambulanten Hilfssystemen. Unterstützte Entscheidung hat Vorrang vor stellvertretender Entscheidung. Der allgemeine Kommentar zur UN-BRK von 2014 fordert den Aufbau von Systemen unterstützter Entscheidung und die Abschaffung von Systemen der stellvertretenden Entscheidung. Der Staatenbericht zu D von 2015 äußert Besorgnis über die Unvereinbarkeit des deutschen Betreuungsrechts mit der Konvention. Daraus ergibt sich ein dringender Bedarf nach Überarbeitung des Betreuungsrechts. Die Konvention ist allerdings heute schon geltendes Recht, insofern gilt es schon jetzt bei der Erstellung von Betreuungsgutachten auf die Konvention Rücksicht zu nehmen. (Zinkler, M [2015] Anforderungen an Gutachten im Betreuungsverfahren nach der UN-Konvention über die Rechte von Menschen mit Behinderungen. In: Recht \& Psychiatrie 33: 67-71)
Dr. Andreas Küthmann, Memmingen, berichtet über die aktuelle Diskussion zum assistierten Suizid und zur Sterbehilfe. Assistierter Suizid ist in D, einigen anderen Ländern und Staaten der USA legal, aber illegal in vielen anderen Ländern. Die Situation in den Niederlanden und Oregon wird erläutert. Im Gesetz der NL ist Einwilligungsfähigkeit Voraussetzung für assistierten Suizid, trotzdem wurden 1000 Patienten ohne Einwilligung getötet (Beine, pers. Mitteilung). Auch in Oregon ist die Einwilligungsfähigkeit Voraussetzung. Seitdem es das Gesetz gibt, steigt die Anzahl kontinuierlich an. 0,2\% der Todesfälle in Oregon sind inzwischen assistierte Suizide. In der Schweiz sind es im Jahr etwa 400 Fälle. Ein Drittel sind nicht schwer körperlich erkrankt. Mexiko wird von USBürgern aufgesucht, weil es dort Pentobarbital im Veterinärhandel frei zu kaufen gibt. Die Musterberufsordnung für Ärzte in D verbietet assistierten Suizid, das gilt aber nicht für alle Landesärztekammern (z.B. BW und Bay, die nur Beistand für Sterbende vorschreiben). Inzwischen gibt es Stellungnahmen der DGPPN und der DGSP zum Thema.

Dr. med. Martin Zinkler, Heidenheim 